Acetyldihydrophenanthridin, $\mathrm{C}_{13} \mathrm{H}_{10}=\mathrm{N}-\mathrm{CO}-\mathrm{CH}_{3}$.

Diese Verbindung wurde erhalten durch Erwärmen der Base mit Essigsäureanhydrid im Einschlufsrohr auf $200^{\circ}$ während einiger Stunden. Der Röbreninhalt wurde in Alkohol gelöst und die Acetylverbinduug daraus durch Wasser in Form öliger Tropfen ausgefällt, welche nach einiger Zeit zu dicken, farblosen Prismen erstarrten. Schmelzpunkt $108^{\circ}$.

$0,1150 \mathrm{~g}$ gaben $0,3390 \mathrm{CO}_{q}$ und $0,0611 \mathrm{H}_{q} \mathrm{O}$.

$\begin{array}{ccc} & \text { Berechnet für } & \text { Gefunden } \\ & \mathrm{C}_{\mathbf{1 5}} \mathrm{H}_{\mathbf{1 3}} \mathrm{NO} & \\ \mathrm{C} & 80,72 & 80,40 \\ \mathrm{H} & 5,83 & 5,90 .\end{array}$

II. Ueber Methylphenanthridine und Chrysidine; von Amé Pictet und S. Erlich.

Der in der vorstehenden Mittheilung besprochene leichte Uebergang des Benzylidenanilins in Phenanthridin durch pyrogene Condensation liefs es wünschenswerth erscheinen, auch andere, dem Benzylidenanilin ähulich constituirte Körper derselben Reaction zu unterwerfen, um so zu anderen, dem Phenanthridin verwandten Basen zu gelangen.

Wir haben uns bis jetzt mit den Benzylidenverbindungen des 0 - und p-Toluidins und der beiden Naphtylamine in dieser Beziehung beschäftigt, und in allen vier Fällen Basen daraus erhalten, welche mit dem Phenanthridin die gröfste Analogie zeigen.

So lieferten Benzyliden-o- und p-toluidin zwei isomere Körper der Formel $\mathrm{C}_{14} \mathrm{H}_{11} \mathrm{~N}$, die als Ortho- und Paramethylphenanthridin aufgefafst werden müssen.

Der Versuch mit dem Benzyliden-0-toluidin bot in dieser Hinsicht ein besonderes Interesse dar, da dieser Körper 
bereits vor mehreren Jahren der Gegenstand einer analogen Untersuchung von Eta $\mathbf{r}^{*}$ ) gewesen ist. Beim Durchleiten seiner Dämpfe durch ein bis zur schwachen Rothgluth erhitztes Verbrennungsrohr erhielt E tard einen Körper der Formel $\mathrm{C}_{14} \mathrm{H}_{11} \mathrm{~N}$, den er für das 0-Methylphenanthridin hielt. Der Eine von uns hat vor einigen Jahren **) bewiesen, dafs diese Auffassung der Constitution des Körpers eine irrige war, und dafs unter den angegebenen Temperaturverhältnissen die Condensation des Benzyliden-o-toluidins in der Weise stattfindet, dafs die neue Kohlenstoffbindung nicht zwischen den beiden Phenylresten, sondern zwischen dem Methyl und der CH-Gruppe des Benzyliden-o-toluidins zu Stande kommt, also im Sinne der Gleichung II und nicht der Gleichung I :

$$
\begin{aligned}
& \text { I. } \underset{\mathrm{C}_{8} \mathrm{H}_{5}-\mathrm{CH}}{\| \mathrm{CH}_{3}-\mathrm{C}_{6} \mathrm{H}_{4}-\mathrm{N}}=2 \mathrm{H}+\stackrel{\mathrm{CH}_{3}-\mathrm{C}_{8} \mathrm{H}_{8}-\mathrm{N}}{\prod_{\mathrm{C}_{6}}-\mathrm{CH}}
\end{aligned}
$$

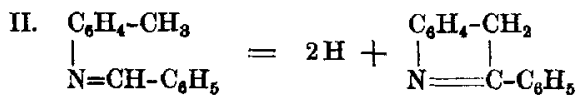

$$
\begin{aligned}
& ={\stackrel{\mathrm{NH}}{\mathrm{C}}-\mathrm{C}-\mathrm{C}_{6} \mathrm{H}_{6} \mathrm{H}_{4}-\mathrm{CH}}_{\|} .
\end{aligned}
$$

Der Etard'sche Körper erwies sich nämlich als identisch ınit dem durch Reduction des o-Nitrodesoxybenzoïns erhaltenen $\alpha$-Phenylindol.

Es war indessen die Möglichkeit nicht ausgeschlossen, dafs bei erhöhter Temperatur die Condensation des Benzylideno-toluidins auch im Sinne der Gleichung I geschehen würde, und in der That konnten wir bei Anwendung einer bis zur hellen Rothgluth erhitzten und mit Bimssteinstücken gefültten Röhre, neben viel Phenylindol, eine beträchtliche Menge o-Methylphenanthridin bekommen.

Durch pyrogene Condensation der beiden Benzyliden-

*) Etard, Comptes rendus \$5, 730 .

**) A mé Pictet, Ber. d. deutseh. chem. Ges. 19, 1063. 
naphtylamine haben wir zwei isomere Basen der Formel $\mathrm{C}_{17} \mathrm{H}_{11} \mathrm{~N}$ erhalten, deren Eigenschaften und Verhalten denen des Phenanthridins vollkommen entsprechen. Sie verdanken offenbar ihre Entstehung einer ähnlichen Reaction, welche folgendermafsen ausgedrückt werden mufs :

$$
\underset{\mathrm{C}_{10} \mathrm{H}_{7}-\mathrm{N}}{\mathrm{C}_{6} \mathrm{H}_{6}-\mathrm{CH}}=2 \mathrm{H}+{\stackrel{\mathrm{C}_{10} \mathrm{H}_{6}-\mathrm{N}}{\mathrm{C}_{6} \mathrm{H}_{4}-\mathrm{CH}}}_{\|}^{\|} .
$$

Diese Basen verhalten sich also zum Chrysen wie das Phenanthridin zum Phenanthren und möchten wir sie deshalb mit dem gemeinsamen Namen Chrysidine bezeichnen.

Dem $\alpha$-Chrysidin, welches aus dem Benzyliden- $\alpha$-naphtylamin entstammt, kommt offenbar folgende Constitution zu :

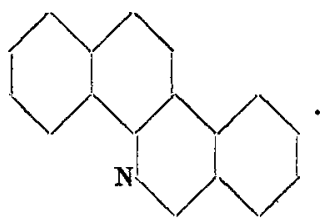

Für das aus dem Benzyliden- $\beta$-naphtylamin entstehende $\beta$-Chrysidin hätte man die Wahl zwischen den zwei folgenden Structurformeln :

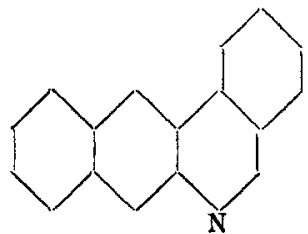

I.

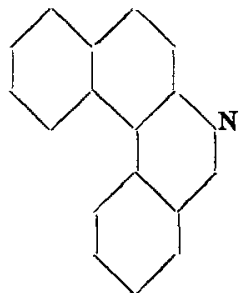

II.

Nach den Erfahrungen, die man im Gebiete der Naphtochinoline gemacht hat, ist es aber höchst wahrscheinlich, dafs die Schliefsung des Pyridinringes hier auch zwischen dem $\beta$ und dem $\alpha$-Kohlenstoff des Naphtalins und nicht zwischen den 
beiden $\beta$-Kohlenstoffatomen erfolgt, dafs also die Formel II der Constitution das $\beta$-Chrysidins entspricht.

Freilich könnte auch ein Gemisch beider Verbindungen zusammen entstehen; es ist uns aber nie gelungen aus dem Condensationsproduct zwei verschiedene Basen zu isoliren; der erhaltene Körper erwies sich immer als ganz einheitlich.

Wie aus den Formeln ersichtlich, stammen die beiden Chrysidine, durch Ersatz einer Methingruppe durch Stickstoff, nicht von demselben Kohlenwasserstoffe $a b$; es liegen ihnen zwei isomere Chrysene zu Grunde. Welches von diesen beiden Isomeren das Steinkohlentheerchrysen repräsentirt, mufs weiteren Untersuchungen über die Constitution dieses Kohlenwasserstoffes überlassen werden, denn weder durch die Synthesen von Graebe und Bungener*) und von Kraemer und Spieker ${ }^{* *}$ ), noch durch die neueren Arbeiten von Bamberger und Burgdor $f^{* * *}$ ), ist noch ein Aufschlufs über die Frage zu ziehen, ob das Chrysen eine dem $\alpha$-oder dem $\beta$-Chrysidin entsprechende Structur besitzt.

Die Gewinnung und Reindarstellung der Methylphenanthridine und der Chrysidine geschah wesentlich nach demselben Verfahren, welches für das Phenanthridin angewandt wurde. Die Benzylidenverhindungen wurden durch ein eisernes, mit Bimssteinstücken gefülltes und bis zur hellen Rothgluth erhitztes Rohr destillirt, das Product von den leichter flüchtigen Körpern (Benzol, Toluol, Benzonitril, Naphtalin) durch Destillation befreit und der Rückstand mit concentrirter Salzsäure in der Wärme behandelt. Nach Verdünnen mit Wasser wurde mit Quecksilberchloridlösung gefällt, der Niederschlag aus verdünnter Salzsäure bis $\mathrm{zu}$ constantem Schmelzpunkt umkrystallisirt und

*) Ber. d. deutsch. chem. Ges. 12, 1078.

**) Daselbst $88,84$.

***) Daselbst $\mathbf{2 3}, \mathbf{2 4 3 3}$. 
daraus durch Schwefelwasserstoff und Natronlauge die Base abgeschieden.

Die Verwandtschaft aller vier Basen mit einander und mit dem Phenanthridin zeigt sich in allen ihren Reactionen und Eigenschaften. Sie sind schwache Basen, deren Salze durch Wasser leicht dissociirt werden. Dieselben sind alle gelb gefärbt; ihre concentrirten Lösungen zeigen eine grüne Fluorescenz, die beim Verdünnen allmählich in's Blaue übergeht.

Als tertiäre Basen vereinigen sie sich leicht mit einem Molekül Jodmethyl zu Körpern, die in Wasser ziemlich leicht, in Alkohol weniger, in Aether nicht löslich sind. Diese Ammoniumjodide werden durch Natronhydrat in der Kälte in krystallisirte Hydroxyde verwandelt, welche in Wasser unlöslich sind, in Alkohol und Aether aber sich leicht mit einer intensiven blauen resp. violetten Fluorescenz lösen. Diese Ammoniumhydroxyde liefern mit Säuren gelbe Salze, deren wässerige Lösungen eine viel stärkere grüne Fluorescenz zeigen als die der ursprünglichen tertiären Basen.

Durch Behandlung mit Zinn und Salzsäure lassen sich die vier Basen leicht zu farblosen, nicht mehr fluorescirenden Hydroderivaten reduciren, welche wir vorläufig nicht näher untersucht haben, von denen wir nur constatirt haben, dafs sie, wie das Dihydrophenanthridin, secundäre Basen sind und Nitrosamine liefern, welche die Li ebermann'sche Reaction zeigen.

Paramethylphenanthridin,

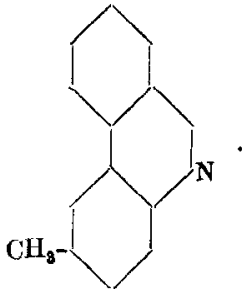

Die aus dem Benzyliden-p-toluidin auf der oben angegebenen Weise gewonnene und vermittelst ihres Quecksilber- 
salzes gereinigte Base krystallisirt aus verdünntem Alkohol in langen, dünnen, weifsen Nadeln, vom Schmelzpunkt 131 ${ }^{\circ}$. Sie ist in Alkohol, Aether, Benzol, Chloroform und Ligroïn leicht löslich, in Wasser sehr wenig. Die alkoholischen und wässerigen Lösungen fluoresciren schwach blau, die ätherische nicht. Mit Wasserdämpfen wird sie schwer übergetrieben. Geruch und Geschmack wie beim Phenanthridin.

$0,1537 \mathrm{~g}$ gaben $0,4885 \mathrm{CO}_{2}$ und $0,0792 \mathrm{H}_{2} \mathrm{O}$.

$0,1462 \mathrm{~g}$, 9,6 cbcm Stickgas bei $10^{\circ}$ und $733 \mathrm{~mm}$ Barometerstand.

$\begin{array}{lcc} & \text { Berechnet für } & \text { Gefunden } \\ \mathrm{C} & \mathrm{C}_{\mathbf{1 4}} \mathrm{H}_{11} \mathbf{N} & \\ \mathrm{H} & \mathbf{8 7 , 0 5} & 86,68 \\ \mathrm{~N} & 5,70 & 5,73 \\ & 7,25 & 7,58 .\end{array}$

Das Chlorhydrat ist in Wasser leicht löslich und krystallisirt beim Erkalten der stark eingeengten Lösung in flachen, hellgelben Nadeln. Beim langsamen Verdunsten über Schwefelsäure wurde es auch in länglichen, sechsseitigen Blättchen erhalten.

Das Sulfat und Nitrat sind ebenfalls in Wasser leicht löslich.

Das Platindoppelsalz, $\left(\mathrm{C}_{14} \mathrm{H}_{11} \mathrm{~N} . \mathrm{HCl}\right)_{2} \mathrm{PtCl}_{4}+2 \mathrm{H}_{2} \mathrm{O}$, ist in verdünnter Salzsäure und in Alkohol auch in der Wärme sehr schwer löslich. Durch heifses Wasser wird es zersetzt und kann daraus nicht umkrystallisirt werden. Durch vorsichtigen Zusatz von Platinchlorid zu einer warmen Lösung des Chlorhydrats kann man es beim Erkalten in Form gelber verzweigter Nadeln bekommen, die bei $280^{\circ}$ noch nicht schmelzen. Das Salz enthält zwei Moleküle Krystallwasser, die bei $110^{\circ}$ entweichen.

$0,4575 \mathrm{~g}$ lufttrockene Substanz verloren bei $110^{\circ} 0,0203 \mathrm{H}_{8} \mathrm{O}$

$$
\begin{array}{ccr} 
& \text { Berechnet für } & \text { Gefunde } \\
\mathrm{H}_{2} \mathrm{O} & \left(\mathrm{C}_{14} \mathrm{H}_{\mathrm{t}} \mathrm{N} . \mathrm{HCl}\right)_{2} \mathrm{PtCl}+2 \mathrm{H}_{2} \mathrm{O} & \\
4,33 & 4,43 .
\end{array}
$$


$0,4372 \mathrm{~g}$ wasserfreie Substanz gaben $0,1052 \mathrm{Pt}$.

$\begin{array}{ccc} & \text { Berechnet für } & \text { Gefunden } \\ & \left(\mathrm{C}_{\mathbf{1}} \mathrm{H}_{\mathbf{1 1}} \mathrm{N} . \mathrm{HCl}\right)_{\mathbf{2}} \mathbf{P t C l} & \\ \mathbf{P t} & \mathbf{2 4 , 4 6} & \mathbf{2 4 , 0 6 .}\end{array}$

Das Quecksilberdoppelsalz wird aus verdünnter Salzsāure in langen, flachen, goldgelben Nadeln erhalten, die sich oft sternförmig gruppiren. In kaltem Wasser ist es wenig mit grüner Fluorescenz löslich und schmilzt bei $215^{\circ}$.

Das Golddoppelsalz ist in Wasser fast unlöslich; es löst sich leicht in heifser verdünnter Salzsäure und scheidet sich daraus beim Erkalten in langen, feinen, hellgelben Nadeln ab. Es schmilzt, nach vorheriger theilweiser Zersetzung, bei $210^{\circ}$.

Das Zinkdoppelsalz bildet, aus salzsäurehaltigem Wasser umkrystallisirt, gelbe, büschelförmig gruppirte Nadeln.

Das Pikrat wird durch Versetzen der salzsauren Lösung der Base mit einer wässerigen Lösung von Pikrinsäure und Umkrystallisiren des Niederschlags aus Alkohol in Form sternoder büschelförmig gruppirter, gelber Nadeln erhalten. Es erleidet schon bei $185^{\circ}$ eine theilweise Zersetzung und schmilzt vollständig bei $202^{\circ}$.

Das Bichromat krystallisirt aus seiner heifsen, wässerigen Lösung in rothgelben, mikroscopischen Nadeln.

Das Jodmethylat, $\mathrm{C}_{14} \mathrm{H}_{11} \mathrm{~N} . \mathrm{CH}_{3} \mathrm{~J}$, wurde durch Krystallisation aus warmem Alkohol, worin es in der Kälte ziemlich schwer löslich ist, in Form kleiner, brauner Nadeln erhalten. Es schmilzt unter Zersetzung bei $180^{\circ}$. Seine gelbe, grün fluorescirende wässerige Lösung wird durch Zusatz einiger Tropfen Kalilauge entfärbt, unter Abscheidung eines voluminösen Niederschlags des Methylhydroxyds, $\mathrm{C}_{14} \mathrm{H}_{11} \mathrm{~N} . \mathrm{CH}_{3} . \mathrm{OH}$. Dasselbe stellt, aus verdünntem Alkohol umkrystallisirt, lange, weirse Nadeln dar, deren Schmelzpunkt bei $136^{\circ}$ liegt. Es ist in Alkohol und Aether leicht löslich, in Wasser fast gar nicht. Die alkoholische Lösung zeigt intensiv blaue, die ätherische violette Fluorescenz. 
Das Methylhydroxyd löst sich in Salzsäure mit grüner Fluorescenz; nach Concentration der Lōsung krystallisirt das Chlormethylat in hellgelben Nadeln, die in kaltem Wasser ziemlich schwer löslich sind. Seine Lösung liefert mit Platinund Quecksilberchlorid Niederschläge der betreffenden Doppelsalze, welche aus kleinen gelben Nadeln bestehen.

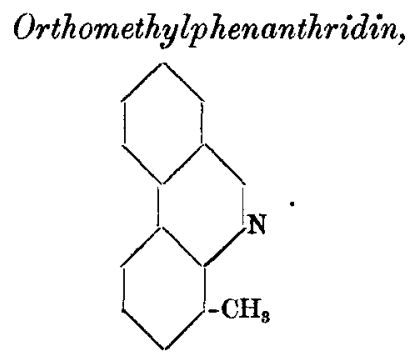

Die Wirkung der Glühhitze auf die Dämpfe des Benzyliden-o-toluidins liefert hauptsächlich $\alpha$-Phenylindol, auch wenn man die Operation bei einer der Weifsgluth nahe liegenden Temperatur ausführt. Neben diesem Körper konnten wir aber doch eine gewisse Menge o-Methylphenanthridin isoliren.

Die Trennung beider Verbindungen geschah durch Lösen der höchstsiedenden Fraction des Destillationsproducts in heifser concentrirter Salzsäure und Eingiefsen in kaltes Wasser. Seinen sehr schwachen basischen Eigenschaften entsprechend wird dabei das Phenylindol vollständig niedergeschlagen, während das 0-Methylphenanthridin, mit wenig Toluidin gemengt, in Lösung bleibt. Die Reinigung wurde, wie bei den übrigen Basen, mit Hülfe des Quecksilbersalzes vorgenommen, wurde aber dadurch erschwert, dafs das 0-Methylphenanthridin ein sehr geringes Krystallisationsvermögen besitzt. Aus sauren Lösungen wird es durch Natron in Form eines Oeles abgeschieden, welches in Berührung mit Wasser nicht zum Erstarren zu bringen ist. Es wurde deshalb in Aether aufgenommen, die Lösung über festem Kali scharf getrocknet und 
eingedampft. Der so erbaltene, ölige Rückstand erstarrte dann allmäblich in einer Kältemischung zu einer halbfesten, krystallinischen Masse, welche durch Auflegen auf eine Thonplatte von den anhängenden, flüssigen Beimischungen so gut wie möglich befreit wurde. Alle unsere Versuche, die so erhaltene Base durch Umkrystallisiren reiner zu bekommen, scheiterten, da dieselbe sich aus allen angewandten Lösungsmitteln wieder ölig abschied. Auch war die zur Verfügung stehende Menge Substanz zu klein, um an eine weitere Reinigung durch Destillation oder durch nochmaliges Ueberführen in ein gut krystallisirendes Salz zu denken. Wir mufsten uns also begnügen, sie in diesem Zustande der Analyse zu unterwerfen :

$0,1914 \mathrm{~g}$ gaben $0,6068 \mathrm{CO}_{2}$ und $0,0981 \mathrm{H}_{8} \mathrm{O}$.

$0,2295 \mathrm{~g} n 16,2$ cbem Stickgas bei $17^{\circ}$ und $720 \mathrm{~mm}$ Barometerstand.

$\begin{array}{lcc} & \text { Berechnet für } & \text { Gefunden } \\ & \mathrm{C}_{\mathbf{1 4}} \mathbf{H}_{11} \mathbf{N} & \\ \mathrm{C} & 87,05 & 86,47 \\ \mathrm{H} & 5,70 & 5,69 \\ \mathrm{~N} & 7,25 & 7,74 .\end{array}$

Der Schmelzpunkt der so erhaltenen Base wurde bei etwa $70^{\circ}$ beobachtet.

Das o-Methylphenanthridin ist in Alkohol, Aether, Chloroform, Benzol und Ligroin sehr leicht löslich. In Berührung mit Wasser verflüssigt es sich sofort, löst sich aber nicht darin. Es besitzt einen schwachen, dem Phenanthridin ähnlichen Geruch; seine Dămpfe sind stechend und reizen zum Niesen.

Das Chlorhydrat krystallisirt aus concentrirter, wässeriger Lösung in feinen, gelben, pinselförmig angeordneten Nadeln. Dieses Salz, sowie das Nitrat, sind in kaltem Wasser leicht löslich und zeigen in verdünnter Lösung eine blaue Fluorescenz. Das Platindoppelsalz scheidet sich aus warmer, verdünnter Annslen der Chemio 266. Bd. 
Salzsäure, in Form hellgelber, dünner Nadeln aus, die bei $275^{\circ}$ noch nicht schmelzen. Seine Zusammensetzung entspricht der Formel $\left(\mathrm{C}_{14} \mathrm{H}_{11} \mathrm{~N} . \mathrm{HCl}\right)_{2} \mathrm{PtCl}_{4}+2 \mathrm{H}_{2} \mathrm{O}$.

$0,1503 \mathrm{~g}$ lufttrockenes Salz verloren bei $110^{\circ} 0,0065 \mathrm{H}_{\mathbf{g}} \mathrm{O}$.

$\begin{array}{ccc} & \text { Berechnet für } & \text { Gefunden } \\ \mathrm{H}_{8} \mathrm{O} & \left(\mathrm{C}_{16} \mathrm{H}_{11} \mathrm{~N} . \mathrm{HCl}\right)_{2} \mathrm{PtCl}_{4}+2 \mathrm{H}_{9} \mathrm{O} & \\ 4,33 & 4,32 .\end{array}$

0,1438 g getrocknetes Salz gaben $0,0353 \mathrm{Pt}$.

$\begin{array}{ccc}\text { Berechnet für } & \text { Gefunden } \\ \mathbf{P t} & \left(\mathrm{C}_{\mathbf{4}} \mathbf{H}_{\mathbf{1 1}} \mathbf{N} . \mathbf{H C l}\right)_{\mathbf{2}} \mathbf{P t C l} & \\ 24,46 & \mathbf{2 4 , 5 4 .}\end{array}$

Das Quecksilberdoppelsalz bildet, aus verdünnter Salzsäure umkrystallisirt, lange, hellgelbe, glänzende Nadeln vom Schmp. $196^{\circ}$.

Das Golddoppelsalz schmilzt bei 196 bis $200^{\circ}$ und wird durch Krystallisation aus verdünnter Salzsäure in feinen, hellgelben, gebogenen Nadeln erhalten.

Das Zinkdoppelsalz stellt lange, in Büscheln gruppirte, gelbe Nadeln dar.

Das Pikrat löst sich leicht in warmem Alkohol uud schiefst daraus beim Erkalten in langen, gelben Nadeln an, die bei $220^{\circ}$ unter Zersetzung schmelzen.

Das Bichromat krystallisirt aus heifsem Wasser in kleinen, orangerothen, federartig gruppirten Nadeln.

Das Jodmethylat, $\mathrm{C}_{14} \mathrm{H}_{11} \mathrm{~N} . \mathrm{CH}_{3} \mathrm{~J}$, bildet, aus Alkohol umkrystallisirt, kleine, braungelbe Nadeln, die bei $187^{\circ}$ unter Zersetzung schmelzen. Es ist in Alkohol und Wasser leicht, in Aether sehr wenig löslich. In verdünnten Lösungen zeigt es eine grüne Fluorescenz.

Durch Zusatz von Natronlauge zu der wässerigen Lösung des Jodmethylats wird das entsprechende Ammoniumhydroxyd ölig gefällt. Es ist uns nicht gelungen, es im krystallisirten Zustande zu erhalten. Es ist in Alkohol und Aether mit einer prachtvoll blauen Fluorescenz löslich, in Wasser sehr wenig. 
In verdünnten Säuren löst es sich mit grüner Fluorescenz. Die salzsaure Lösung liefert durch Quecksilberchlorid einen aus kleinen, büschelförmig gruppirten Nadeln bestehenden Niederschlag.

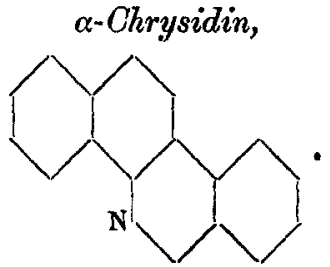

Die Darstellung des $\alpha$-Chrysidins aus dem Benzyliden- $\alpha-$ naphtylamin geschah wesentlich auf dieselbe Weise wie die der anderen Basen; nur ist hier die Ausbeute beträchtlich besser und wird auch die Reingewinnung der Base durch den Umstand sehr erleichtert, dafs ihr salzsaures Salz in Wasser sehr wenig löslich ist. Die Reinigung durch das Quecksilbersalz kann hier deshalb vermieden werden und es genügt, die über $300^{\circ}$ siedenden Antheile des Condensationsproductes mit concentrirter Salzsäure zu digeriren und die Lösung in kaltes Wasser zu giefsen, wobei das Chlorhydrat des $\alpha$-Chrysidins als voluminöser, flockiger, gelber Niederschlag ausfällt. Derselbe wird aus heifser verdünnter Salzsäure umkrystallisirt und mit Natron zersetzt. Die aus Alkohol umkrystallisirte Base lieferte bei der Analyse, nach dem Trocknen über Schwefelsäure, folgende auf die Formel $\mathrm{C}_{17} \mathrm{H}_{11} \mathrm{~N}$ passende Werthe : $0,1644 \mathrm{~g}$ gaben $0,5349 \mathrm{CO}_{x}$ und $0,0747 \mathrm{H}_{2} \mathrm{O}$.

$0,1634 \mathrm{~g}$ " $9 \mathrm{cbcm}$ Stickgas bei $15^{0}$ und $736 \mathrm{~mm}$ Barometerstand.

$\begin{array}{ccc} & \text { Berechnet für } & \text { Gefunden } \\ & \mathrm{C}_{\mathbf{1 7}} \mathbf{H}_{\mathbf{1 1}} \mathrm{N} & \\ \mathrm{C} & 89,08 & 88,74 \\ \mathrm{H} & \mathbf{4 , 8 0} & \mathbf{5 , 0 4} \\ \mathrm{N} & \mathbf{6 , 1 2} & 6,24 .\end{array}$

Aus verdünntem Alkohol umkrystallisirt, bildet das 
$\alpha$-Chrysidin lange, haarfeine, weifse Nadeln, die bei $108^{\circ}$ schmelzen. In absolutem Alkohol ist es leicht löslich und wird bei freiwilligem Verdunsten des Losungsmittels in langen, flachen Nadeln erhalten. In Benzol, Aether, Chloroform und Ligroïn löst es sich ebenfalls leicht, in Wasser gar nicht. Die alkoholische Lösung fluorescirt blau.

Das $\alpha$-Chrysidin ist eine sehr schwache Base, deren Salze durch Wasser schon in der Kälte zersetzt werden und nur bei Gegenwart freier Säure beständig sind. Ihre Lösungen sind gelb und zeigen eine schöne, blaugrüne Fluorescenz.

Das Chlorhydrat krystallisirt aus heifser, verdünnter Salzsäure in gelben Nadeln, die sich stern- oder büschelförmig gruppiren; es schmilzt bei etwa $210^{\circ}$.

Das Nitrat ist in kaltem Wasser noch schwerer löslich als das salzsaure Salz und wird aus der Lösung desselben durch Zusatz von Salpetersäure als flockiger Niederschlag gefällt. Durch Umkrystallisiren aus warmem, salpetersäurehaltigem Wasser wird es in Form gelber, büschelförmig vereinigter Prismen erhalten, deren Schmelzpuukt bei $155^{\circ}$ liegt.

Das Platindoppelsalz, $\left(\mathrm{C}_{17} \mathrm{H}_{11} \mathrm{~N} . \mathrm{HCl}\right)_{2} \mathrm{PtCl}_{4}+2 \mathrm{H}_{2} \mathrm{O}$, krystallisirt aus verdüınter Salzsäure in langen, gelben, verästelten. Nadeln, die sich bei $255^{\circ}$ zersetzen.

$0,1609 \mathrm{~g}$ lufttrockenes Salz verloren bei $110^{\circ} 0,0060 \mathrm{H}_{2} \mathrm{O}$.

$$
\begin{array}{ccc} 
& \text { Berechnet für } & \text { Gefunden } \\
\mathrm{H}_{2} \mathrm{O} & \left(\mathrm{C}_{17} \mathrm{H}_{11} \mathrm{~N} . \mathrm{HCl}\right)_{2} \mathrm{PtCl}{ }_{4}+2 \mathrm{H}_{2} \mathrm{O} & \\
& 3,99 & \mathbf{3}, \mathbf{7 3} .
\end{array}
$$

$0,2956 \mathrm{~g}$ wasserfreies Salz gaben $0,0660 \mathrm{Pt}$.

$\begin{array}{ccc} & \text { Berechnet für } & \text { Gefunden } \\ \text { Pt } & \left(\mathrm{C}_{\mathbf{1 7}} \mathrm{H}_{11} \mathrm{~N} . \mathrm{HCl}\right)_{\mathbf{2}} \mathrm{PtCl} & \\ & 22,42 & 22,33 .\end{array}$

Das Quecksilberdoppelsalz wird durch Versetzen der salzsauren Lösung der Base mit Sublimatlösung und Umkrystallisiren aus verdünnter Salzsäure in langen, gelben, gebogenen Nadein erhalten, die bei 240 bis $245^{\circ}$ schmelzen. 
Das Golddoppelsalz bildet hellgelbe, büschelförmig gruppirte Nadeln vom Schmelzpunkt 2280, das Zinkdoppelsalz lange, gelbe Nadeln, die gegen $250^{\circ}$ schmelzen, das Bichromat kleine, sternförmig gruppirte Nadeln.

Das Pikrat entsteht durch Vermischen von Lösungen der Base und Pikrinsäure in Benzol. Aus Alkohol krystallisirt es in langen, gelben Nadeln und schmilzt bei $240^{\circ}$.

Das Jodmethylat, $\mathrm{C}_{17} \mathrm{H}_{11} \mathrm{~N} . \mathrm{CH}_{3} \mathrm{~J}$, wird erhalten durch zweistündiges Erhitzen der Base mit Jodmethyl im Einschlufsrohr auf $100^{\circ}$ und Umkrystallisiren aus Alkohol unter Zusatz von Thierkoble in Form hellgelber, sternförmig gruppirter Nadeln, die bei $108^{\circ}$ schmelzen. Es ist in kaltem Alkohol wenig löslich, in heifsem leichter. In Aether ist es unlöslich.

Durch Natronlauge wird die wässerige Lösung des Jodmethylats gefällt; der entstehende weifse Niederschlag wurde in Alkohol aufgelöst und zu der Lösung langsam Wasser zugegeben. Es erfolgte augenblicklich eine reichliche Ausscheidung des Methylhydroxyds $\left(\mathrm{C}_{17} \mathrm{H}_{11} \mathrm{~N} \mathrm{CH}_{3} . \mathrm{OH}\right)$ in Form schöner, glänzender, weifser Nadeln, deren Schmelzpunkt bei $110^{\circ}$ liegt. Dieser Körper ist in kaltem Alkohol wenig, in Aether leicht löslich. Von Wasser wird er, auch in der Wärme, nur spurenweise gelöst. Die Lösungen in Alkohol und Aether zeigen eine intensive blaue resp. violette Fluorescenz.

Durch Salzsäure wird das Methylhydroxyd in das schwerlösliche Chlormethylat übergeführt, welches aus verdūnnter Salzsäure in langen, feinen Nadeln auskrystallisirt. Die wässerige, stark grün fluorescirende Lösung des Salzes giebt mit Quecksilberchlorid ein bei $215^{\circ}$ schmelzendes Quecksilberdoppelsalz, und mit Platinchlorid ein in kleinen gelben Nadeln krystallisirendes Platindoppelsalz. Dasselbe ist krystallwasserfrei und ergab bei der Analyse folgenden Platingehalt :

0,1208 g gaben 0,0262 Pt.

$$
\text { Pt } \begin{array}{cc}
\text { Berechnet für } & \text { Gefunden } \\
\left(\mathrm{C}_{17} \mathrm{H}_{11} \mathrm{~N} . \mathrm{CH}\right. & \mathrm{Cl})_{2} \mathrm{PtCl}_{5} \\
21,73 & 21,69 .
\end{array}
$$




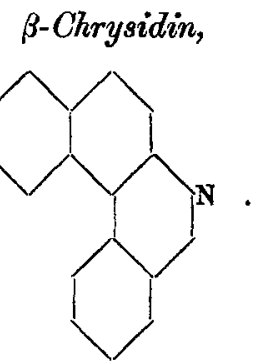

Die aus Benzyliden- $\beta$-naphtylamin entstehende und durch das Quecksilberdoppelsalz gereinigte Base bildet, aus warmem, verdünntem Alkohol umkrystallisirt, silberglänzende, weifse Nadeln vom Schmelzpunkt 1310. Ihre Zusammensetzung entspricht der Formel $\mathrm{C}_{17} \mathrm{H}_{11} \mathrm{~N}$.

$0,1218 \mathrm{~g}$ gaben $0,3968 \mathrm{CO}_{\mathrm{g}}$ und $0,0540 \mathrm{H}_{2} \mathrm{O}$.

$0,0799 \mathrm{~g} \cap 4,5$ cbem Stickgas bei $14^{\circ}$ und $721 \mathrm{~mm}$ Barometerstand.

$\begin{array}{ccc} & \begin{array}{c}\text { Berechnet für } \\ \mathrm{C}_{17} \mathrm{H}_{11} \mathrm{~N}\end{array} & \text { Gefunden } \\ \mathrm{C} & 89,08 & 88,85 \\ \mathrm{H} & 4,80 & 4,93 \\ \mathrm{~N} & 6,12 & 6,42 .\end{array}$

Das $\beta$-Chrysidin ist in seinen Eigenschaften und Verhalten dem $\alpha$-Isomeren sehr ähnlich. Löslichkeitsverhältnisse und Fluorescenzerscheinungen sind dieselben. Die Salze besitzen etwas höhere Schmelzpunkte und werden durch Wasser noch leichter dissociirt.

Das Chlorhydrat ist in Wasser leichter löslich als das entsprechende Salz des $\alpha$-Chrysidins. Es krystallisirt aus seiner concentrirten wässerigen Lösung in kleinen, gelben, wohlausgebildeten Prismen, welche dem orthorhombischen System angehören und gegen $220^{\circ}$ schmelzet.

Das Nitrat ist schwer löslich und wird aus der Lösung des Chlorhydrats durch Salpetersäure gefällt. Aus warmem, salpetersäurehaltigem Wasser scheidet es sich beim Erkalten 
in kugeligen Aggregaten ab, die aus kleinen gelben Nadeln bestehen. Schmelzpunkt $187^{\circ}$.

Das Platindoppelsalz, $\left(\mathrm{C}_{17} \mathrm{H}_{11} \mathrm{~N} . \mathrm{HCl}\right)_{2} \mathrm{PtCl}_{4}+2 \mathrm{H}_{2} \mathrm{O}$, bildet, aus verdünnter Salzsäure umkrystallisirt, hellgelbe mikroskopische Nadeln, die bei $245^{\circ}$ unter Zersetzung schmelzen.

$0,2136 \mathrm{~g}$ lufttrockenes Salz verloren bei $110^{\circ} 0,0092 \mathrm{H}_{2} \mathrm{O}$.

$$
\mathrm{H}_{2} \mathrm{O} \quad \begin{array}{cc}
\text { Berechnet für } & \text { Gefunden } \\
\left(\mathrm{C}_{17} \mathrm{H}_{11} \mathrm{~N} . \mathrm{HCl}\right)_{2} \mathrm{PtCl} \\
\mathbf{3 , 9 9}+2 \mathrm{H}_{2} \mathrm{O} & \\
& 3,30 .
\end{array}
$$

$0,2044 \mathrm{~g}$ wasserfreies Salz gaben $0,0451 \mathrm{Pt}$.

$\begin{array}{ccc} & \text { Berechnet für } & \text { Gefunden } \\ \mathrm{Pt} & \left(\mathrm{C}_{17} \mathrm{H}_{11} \mathrm{~N} \cdot \mathrm{HCl}\right)_{\mathbf{2}} \mathrm{PtCl}_{4} & \\ 22,43 & 22,06 .\end{array}$

Das Bichromat, $\left(\mathrm{C}_{17} \mathrm{H}_{11} \mathrm{~N}\right)_{2} \mathrm{H}_{2} \mathrm{Cr}_{2} \mathrm{O}_{7}+2 \mathrm{H}_{2} \mathrm{O}$, wurde aus heifsem Wasser in orangegelben, büschelförmig gruppirten Nadeln erhalten. Dieses Salz ist in kaltem Wasser sehr wenig löslich und zersetzt sich gegen $200^{\circ}$ ohne zu schmelzen.

$0,1063 \mathrm{~g}$ lufttrockenes Salz verloren bei $110^{\circ} 0,0053 \mathrm{H}_{2} \mathrm{O}$.

$$
\begin{array}{ccc} 
& \text { Berechnet für } & \text { Gefunden } \\
\mathrm{H}_{2} \mathrm{O} & \left(\mathrm{C}_{17} \mathrm{H}_{11} \mathrm{~N}\right)_{2} \mathrm{H}_{1} \mathrm{Cr}_{2} \mathrm{O}_{7}+2 \mathrm{H}_{2} \mathrm{O} & \\
5,06 & 4,99 .
\end{array}
$$

$0,1010 \mathrm{~g}$ wasserfreies Salz gaben $0,0225 \mathrm{Cr}_{8} \mathrm{O}_{3}$, entsprechend $0,0154 \mathrm{Cr}$.

$\begin{array}{ccc} & \begin{array}{c}\text { Berechnet für } \\ \left(\mathrm{C}_{\mathbf{1 7}} \mathrm{H}_{\mathbf{4 1}} \mathrm{N}\right)_{\mathbf{2}} \mathrm{H}_{\mathbf{2}} \mathrm{Cr}_{\mathbf{3}} \mathrm{O}_{7}\end{array} & \text { Gefunden } \\ \mathrm{Cr} & 15,38 & 15,24 .\end{array}$

Es wurden ferner dargestellt :

Das Quecksilberdoppelsalz, hellgelbe Nadeln vom Schmelzpunkt $\mathbf{2 7 2 ^ { 0 }}$, in verdünnter Salzsäure sehr wenig löslich.

Das Golddoppelsalz, feine, schwach gelb gefärbte Nadeln, die bei $245^{\circ}$ nach theilweiser Zersetzung schmelzen. Dieses Salz ist in Wasser viel Jeichter löslich als die Chloraurate der anderen Basen.

Das Zinkdoppelsalz, schöne, gelbe Nadeln. Schmelzpunkt $197^{\circ}$. 
Das Pikrat, kleine, gelbe Körner (aus Alkohol), die aus mikroskopischen Nadeln gebildet sind. In heifsem Wasser ist es fast unlöslich. Es wird bei $210^{\circ}$ dunkel und zersetzt sich vollständig bei $245^{\circ}$.

Das Jodmethylat, $\mathrm{C}_{17} \mathrm{H}_{11} \mathrm{~N} . \mathrm{CH}_{3} \mathrm{~J}$, wird durch Umkrystallisiren aus Alkohol unter Zusatz von Thierkohle in Form brauner glänzender Nadeln erbalten, deren Schmelzpunkt bei 237 ${ }^{\circ}$ liegt. In kaltem Alkohol und Wasser ist es wenig, in den heifsen Flüssigkeiten ziemlich leicht löslich. Aether löst es nicht auf.

In der wässerigen grünfluorescirenden Lösung des Jodmethylats erzeugt Natronlauge eine voluminöse Fällung des Methylhydroxyds. Dasselbe, in warmem Alkohol aufgelöst, scheidet sich beim Erkalten in flachen, weifsen Nadeln vom Schmelzpunkt $133^{\circ}$ aus. Es ist in Alkohol und Aether leicht löslich, in Wasser fast gar nicht. Die alkoholische Lösung zeigt eine blaue, die ätherische eine intensiv violette Fluorescenz.

Durch Behandeln des Methylhydroxyds mit Salzsäure entsteht das schwerlösliche Chlormethylat, welches aus warmer verdünnter Salzsäure in langen, seideglänzenden Nadeln auskrystallisirt. Durch Zusatz von Platinchlorid zu seiner heifsen Lösung wurde das entsprechende Chlorplatinat in Form mikroskopischer gelber Nadeln erhalten. Dieses Salz ist wasserfrei und zeigte folgenden Platingehalt :

$0,0863 \mathrm{~g}$ gaben $0,0186 \mathrm{Pt}$.

$$
\begin{array}{ccc} 
& \begin{array}{c}
\text { Berechnet für } \\
\left(\mathrm{C}_{17} \mathrm{H}_{11} \mathrm{~N} . \mathrm{CH}_{3} \mathrm{Cl}\right)_{\mathbf{2}} \mathrm{PtCl}_{4}
\end{array} & \text { Gefunden } \\
\text { Pt } & 21,73 & 21,55 .
\end{array}
$$

Zum Schlurs seien noch die Schmelzpunkte der fünf bis jetzt dargestellten Phenanthridinbasen und ihrer wichtigsten Salze zur besseren Uebersicht tabellarisch zusammengestellt : 


\begin{tabular}{l|c|c|c|c|c|c}
\hline & $\begin{array}{c}\text { Phenan- } \\
\text { thridin }\end{array}$ & $\begin{array}{c}\text { o-Methyl- } \\
\text { phenan- } \\
\text { thridin }\end{array}$ & $\begin{array}{c}p \text {-Methyl- } \\
\text { phenan- } \\
\text { thridin }\end{array}$ & $\begin{array}{c}\alpha \text {-Chrysi- } \\
\text { din }\end{array}$ & $\begin{array}{c}\beta \text {-Chrysi- } \\
\text { din }\end{array}$ \\
\hline Base. . . . & 104 & gegen 70 & 131 & 108 & 131 \\
Quecksilberdoppel- & & & & & \\
salz.... . . & 197 & 196 & 215 & $240-245$ & 272 \\
Golddoppelsalz. . & 212 & $196-200$ & 210 & 228 & 245 \\
Pikrat... . . & 235 & 220 & 202 & 240 & 245 \\
Jodmethylat. . . & 202 & 187 & 180 & 108 & 237 \\
Methylbydrat . . & 109 & - & 136 & 110 & 133
\end{tabular}

Mittheilung aus dem chemischen Laboratorium der Akademio der Wissenschaften zu München.

\section{Ueber die Constitution des Benzols ;} von Adolf Baeyer.

(Eingelaufen den 4. August 1891.)

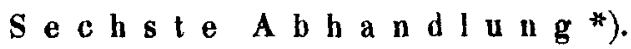

Ueber die Hydronaphtoësäuren.

Als die Untersuchung über die Reductionsproducte der Terephtalsäure nahezu beendet war, liefs ich die Reductionsproducte der $\alpha$ - und $\beta$-Naphtoësäure von den Herren Schoder und Besemfelder in Angriff nehmen, um zu sehen ob bei diesen Säuren sich ähnliche GesetzmäIsigkeiten vorfinden. Diese Arbeiten wurden im Frühjahr 1890 der philosophischen Fakultat der hiesigen Universität als InauguralDissertationen überreicht und erschienen als solche noch in

*) Die 5 ersten Abhandlungen befinden sich in diesen Annalen 245, 103 ; 251, 257 ; 256, 1 ; 258, 1 ; 258, 145. 